\title{
Neutrino Flavor States and the Quantum Theory of Neutrino Oscillations
}

\author{
Carlo Giunti \\ INFN, Sezione di Torino, and Dipartimento di Fisica Teorica, \\ Università di Torino, Via P. Giuria 1, I-10125 Torino, Italy
}

\begin{abstract}
The definition and derivation of flavor neutrino states in the framework of standard Quantum Field Theory is reviewed, clarifying some subtle points. It is shown that a flavor neutrino state that describes a neutrino produced or detected in a charged-current weak interaction process depends on the process under consideration and is appropriate for the description of neutrino oscillations as well as for the calculation of neutrino production or detection rates. Hence, we have a consistent framework for the description of neutrino oscillations and interactions in neutrino oscillation experiments. The standard flavor neutrino states are obtained as approximations which describe neutrinos in experiments that are not sensitive to the dependence of neutrino interactions on the neutrino mass differences. It is also shown that the oscillation probability can be derived either through the usual light-ray time $=$ distance approximation or through an average of the space-time dependent oscillation probability over the unobserved propagation time.
\end{abstract}

To be published as a Topical Review in

Journal of Physics G: Nuclear and Particle Physics

\section{Contents}

Contents

1 Introduction 2

2 Standard Derivation of the Neutrino Oscillation Probability 3

3 Flavor Neutrino States 
5 Neutrino Oscillations

6 Universality of the Oscillation Phases

7 Time Average

8 Conclusions

\section{Introduction}

The standard theory of neutrino oscillations was derived in 1975-76 [1, 2, 3, 4] under the assumption that a flavor neutrino $\nu_{\alpha}$, produced or detected in a charged-current weak interaction process together with a charged lepton with flavor $\alpha(\alpha=e, \mu, \tau)$ is described by the standard flavor state

$$
\left|\nu_{\alpha}\right\rangle=\sum_{k} U_{\alpha k}^{*}\left|\nu_{k}\right\rangle
$$

where $U$ is the unitary mixing matrix of the neutrino fields and $\left|\nu_{k}\right\rangle$ are the Fock states of the massive neutrino fields, with corresponding masses $m_{k}$ (see the reviews in Refs. [5, 6. [7, 8, 9]).

In Ref. [10] it has been shown that the flavor state in Eq. (1.1) is not a quantum of the flavor field $\nu_{\alpha}$ (it is not annihilated by $\nu_{\alpha}$ if the neutrino masses are taken into account). However, it was shown that the flavor state in Eq. (1.1) describes a flavor neutrino $\nu_{\alpha}$ in the realistic ultrarelativistic approximation $\left(m_{k} \ll E\right.$, where $E$ is the neutrino energy) [10].

Later [11], it has been discovered that it is possible to construct a Fock space of flavor states, which allows an alternative description of neutrino oscillations (see also Refs. [12, 13, 14, 15, 16, 17, 18, 19, 20]). However, this construction suffers from mysterious features (the number of flavor Fock spaces is infinite, depending on arbitrary mass parameters; each flavor vacuum and the corresponding ladder operators are timedependent; the ladder operators at different times do not satisfy the canonical anticommutation relations) and it has been shown that the flavor Fock states cannot be applied to the calculation of interaction processes [21, 22].

Let us emphasize that the inapplicability of flavor Fock space theories to the calculation of neutrino interactions is a crucial shortcoming for their applicability to the description of neutrino oscillations, because any oscillation experiment involves the production and detection of neutrinos.

It is then natural to ask if the standard theory of neutrino oscillations is appropriate for the description of neutrino interaction processes. In other words, are the standard flavor states in Eq. (1.1) appropriate for the calculation of the neutrino production and detection rates? 
In this paper we review the derivation the flavor neutrino states in the framework of standard Quantum Field Theory, clarifying some subtle issues. We will show that the flavor neutrino states are appropriate for the description of neutrino production and detection, as well as for the description of neutrino oscillations. 1 . We will see that the flavor neutrino states reduce to the standard flavor states in Eq. (1.1) in the case of experiments which are not sensitive to the difference of the neutrino masses. In this case, we recover the standard oscillation probability.

In this paper we adopt the so-called "plane wave approximation", in which the massive neutrino components of a flavor state are described by plane waves, as in the standard approach (see the reviews in Refs. [5, 6, 7, 8, 9]). However, for the justification of some assumptions in the derivation of the oscillation probability, we will need to take into account the wave packet character of propagating massive neutrinos [25, 26, 27, 28, 29, 30, 31, 32, 33, 34, 35, 36, 37, 38, 39, albeit without need of a specific model.

The plan of the paper is as follows. In section 2 we review the standard derivation of the neutrino oscillation probability, highlighting the underlying assumptions. In section 3 we present a derivation of the flavor neutrino states in the framework of the standard Quantum Field Theory. In section 4 we show that the flavor neutrino states are appropriate for the description of neutrino production and detection. In section 5, using these flavor states, we derive the probability of neutrino oscillations, taking into account the general possibility that different massive neutrinos may have different momenta as well as different energies [40, 27, 41, 42, 33]. In section 6] we show that the corrections to the standard oscillation phase due to violations of the light-ray time $=$ distance approximation and the one-dimensional propagation approximation, adopted in section 5, are negligible. In section 7 we show that the standard oscillation probability can also be derived through the average over the unobserved propagation time of the space-time dependent oscillation probability, taking into account the general properties of the massive neutrino wave packets.

\section{Standard Derivation of the Neutrino Oscillation Probability}

Neutrino oscillations are a consequence of neutrino mixing:

$$
\nu_{\alpha L}(x)=\sum_{k} U_{\alpha k} \nu_{k L}(x) \quad(\alpha=e, \mu, \tau)
$$

where $\nu_{\alpha L}(x)$ are the left-handed flavor neutrino fields, $\nu_{k L}(x)$ are the left-handed massive neutrino fields and $U$ is the unitary mixing matrix (see the reviews in Refs. [5, 6, 7, 8, 9]). Since a flavor neutrino $\nu_{\alpha}$ is created by $\nu_{\alpha L}^{\dagger}(x)$ in a charged-current weak interaction process, in the standard plane-wave theory of neutrino oscillations [1, 2, 3, 4, 5], it is assumed that $\nu_{\alpha}$ is described by the standard flavor state in Eq. (1.1), which has the same mixing as the field $\nu_{\alpha L}^{\dagger}(x)$.

\footnotetext{
${ }^{1}$ In this review we consider neutrino oscillations on vacuum. We do not consider the more complicated case of neutrino oscillations in matter [23, 24].
} 
The massive neutrino states $\left|\nu_{k}\right\rangle$ have definite mass $m_{k}$ and definite energy $E_{k}$. Hence, they evolve in time as plane waves:

$$
i \frac{\partial}{\partial t}\left|\nu_{k}(t)\right\rangle=\mathscr{H}_{0}\left|\nu_{k}(t)\right\rangle=E_{k}\left|\nu_{k}(t)\right\rangle \quad \Longrightarrow \quad\left|\nu_{k}(t)\right\rangle=e^{-i E_{k} t}\left|\nu_{k}\right\rangle
$$

where $\mathscr{H}_{0}$ is the free Hamiltonian operator and $\left|\nu_{k}(t=0)\right\rangle=\left|\nu_{k}\right\rangle$ (all the massive neutrinos start with the same arbitrary phase). The resulting time evolution of the flavor neutrino state Eq. (1.1) is given by

$$
\left|\nu_{\alpha}(t)\right\rangle=\sum_{k} U_{\alpha k}^{*} e^{-i E_{k} t}\left|\nu_{k}\right\rangle=\sum_{\beta=e, \mu, \tau}\left(\sum_{k} U_{\alpha k}^{*} e^{-i E_{k} t} U_{\beta k}\right)\left|\nu_{\beta}\right\rangle .
$$

Hence, if the mixing matrix $U$ is different from unity (i.e. if there is neutrino mixing), the state $\left|\nu_{\alpha}(t)\right\rangle$, which has pure flavor $\alpha$ at the initial time $t=0$, evolves in time into a superposition of different flavors. The quantity in parentheses in Eq. (2.3) is the amplitude of $\nu_{\alpha} \rightarrow \nu_{\beta}$ transitions at the time $t$ after $\nu_{\alpha}$ production. The probability of $\nu_{\alpha} \rightarrow \nu_{\beta}$ transitions at the time $t=T$ of neutrino detection is given by

$$
P_{\nu_{\alpha} \rightarrow \nu_{\beta}}(T)=\left|\left\langle\nu_{\beta} \mid \nu_{\alpha}(T)\right\rangle\right|^{2}=\left|\sum_{k} U_{\alpha k}^{*} e^{-i E_{k} T} U_{\beta k}\right|^{2}=\sum_{k, j} U_{\alpha k}^{*} U_{\beta k} U_{\alpha j} U_{\beta j}^{*} e^{-i\left(E_{k}-E_{j}\right) T} .
$$

One can see that $P_{\nu_{\alpha} \rightarrow \nu_{\beta}}(T)$ depends on the energy differences $E_{k}-E_{j}$. In the standard theory of neutrino oscillations it is assumed that all massive neutrinos have the same momentum $\vec{p}$. Since detectable neutrinos are ultrarelativistid, we have

$$
E_{k}=\sqrt{\vec{p}^{2}+m_{k}^{2}} \simeq E+\frac{m_{k}^{2}}{2 E} \Longrightarrow E_{k}-E_{j}=\frac{\Delta m_{k j}^{2}}{2 E}
$$

where $\Delta m_{k j}^{2} \equiv m_{k}^{2}-m_{j}^{2}$ and $E \equiv|\vec{p}|$ is the energy of a massless neutrino (or, in other words, the neutrino energy in the massless approximation). In most neutrino oscillation experiments the time $T$ between production and detection is not measured, but the source-detector distance $L$ is known. In this case, in order to apply the oscillation probability to the data analysis it is necessary to express $t$ as a function of $L$. Considering ultrarelativistic neutrinos, we have $T \simeq L$, leading to the standard formula for the oscillation probability:

$$
P_{\nu_{\alpha} \rightarrow \nu_{\beta}}(L, E)=\sum_{k, j} U_{\alpha k}^{*} U_{\beta k} U_{\alpha j} U_{\beta j}^{*} \exp \left(-i \frac{\Delta m_{k j}^{2} L}{2 E}\right) .
$$

Summarizing, there are three main assumptions in the standard theory of neutrino oscillations:

(A1) Neutrinos produced or detected in charged-current weak interaction processes are described by the flavor states in Eq. (1.1).

\footnotetext{
${ }^{2}$ It is known that neutrino masses are smaller than about one eV (see the reviews in Refs. [43, 44 ). Since only neutrinos with energy larger than about $100 \mathrm{keV}$ can be detected (see the discussion in Ref. [32]), in oscillation experiments neutrinos are always ultrarelativistic.
} 
(A2) The massive neutrino states $\left|\nu_{k}\right\rangle$ in Eq. (1.1) have the same momentum ("equalmomentum assumption").

(A3) The propagation time is equal to the distance $L$ traveled by the neutrino between production and detection ("time = distance assumption").

In the following we will show that the assumptions (A1) and (A3) correspond to approximations which are appropriate in the analysis of current neutrino oscillation experiments. Instead, the equal-momentum assumption (A2) is not physically justified [40, 27, 41, 42, 33, as one can easily understand from the application of energy-momentum conservation to the production proces 3 . However, in section 5 we will show that the assumption (A2) is actually not necessary for the derivation of the oscillation probability if both the evolutions in space and in time of the neutrino state are taken into account.

\section{Flavor Neutrino States}

The state of a flavor neutrino $\nu_{\alpha}$ is defined as the state which describes a neutrino produced in a charged-current weak interaction process together with a charged lepton $\ell_{\alpha}^{+}$ or from a charged lepton $\ell_{\alpha}^{-}\left(\ell_{\alpha}^{ \pm}=e^{ \pm}, \mu^{ \pm}, \tau^{ \pm}\right.$for $\alpha=e, \mu, \tau$, respectively), or the state which describes a neutrino detected in a charged-current weak interaction process with a charged lepton $\ell_{\alpha}^{-}$in the final state. In fact, the neutrino flavor can only be measured through the identification of the charged lepton associated with the neutrino in a charged-current weak interaction process.

Let us first consider a neutrino produced in the generic decay process

$$
\mathrm{P}_{\mathrm{I}} \rightarrow \mathrm{P}_{\mathrm{F}}+\ell_{\alpha}^{+}+\nu_{\alpha}
$$

where $\mathrm{P}_{\mathrm{I}}$ is the decaying particle and $\mathrm{P}_{\mathrm{F}}$ represents any number of final particles. For example: in the pion decay process

$$
\pi^{+} \rightarrow \mu^{+}+\nu_{\mu}
$$

we have $\mathrm{P}_{\mathrm{I}}=\pi^{+}, \mathrm{P}_{\mathrm{F}}$ is absent and $\alpha=\mu$; in a nuclear $\beta^{+}$decay process $\mathrm{N}(A, Z) \rightarrow$ $\mathrm{N}(A, Z-1)+e^{+}+\nu_{e}$ we have $\mathrm{P}_{\mathrm{I}}=\mathrm{N}(A, Z), \mathrm{P}_{\mathrm{F}}=\mathrm{N}(A, Z-1)$ and $\alpha=e$. The following method can easily be modified in the case of a $\nu_{\alpha}$ produced in the generic scattering process $\ell_{\alpha}^{-}+\mathrm{P}_{\mathrm{I}} \rightarrow \mathrm{P}_{\mathrm{F}}+\nu_{\alpha}$ by replacing the $\ell_{\alpha}^{+}$in the final state with a $\ell_{\alpha}^{-}$in the initial state.

The final state resulting from the decay of the initial particle $P_{I}$ is given by

$$
|f\rangle=\mathrm{S}\left|\mathrm{P}_{\mathrm{I}}\right\rangle
$$

where $S$ is the $S$-matrix operator. Since the final state $|f\rangle$ contains all the decay channels of $\mathrm{P}_{\mathrm{I}}$, it can be written as

$$
|f\rangle=\sum_{k} \mathcal{A}_{\alpha k}^{\mathrm{P}}\left|\nu_{k}, \ell_{\alpha}^{+}, \mathrm{P}_{\mathrm{F}}\right\rangle+\ldots
$$

\footnotetext{
${ }^{3}$ A different opinion, in favor of the equal-momentum assumption, has been recently expressed in Ref. 45]. On the other hand, other authors [46, 47, 48, advocated an equal-energy assumption, which we consider as unphysical as the equal-momentum assumption.
} 
where we have singled out the decay channel in Eq. (3.1) and we have taken into account that the flavor neutrino $\nu_{\alpha}$ is a coherent superposition of massive neutrinos $\nu_{k}$. Since the states of the other decay channels represented by dots in Eq. (3.4) are orthogonal to $\left|\nu_{k}, \ell_{\alpha}^{+}, \mathrm{P}_{\mathrm{F}}\right\rangle$ and the different states $\left|\nu_{k}, \ell_{\alpha}^{+}, \mathrm{P}_{\mathrm{F}}\right\rangle$ are orthogonal and normalized, the coefficients $\mathcal{A}_{\alpha k}^{\mathrm{P}}$ are the amplitudes of production of the corresponding state in the decay channel in Eq. (3.1):

$$
\mathcal{A}_{\alpha k}^{\mathrm{P}}=\left\langle\nu_{k}, \ell_{\alpha}^{+}, \mathrm{P}_{\mathrm{F}} \mid f\right\rangle=\left\langle\nu_{k}, \ell_{\alpha}^{+}, \mathrm{P}_{\mathrm{F}}|\mathrm{S}| \mathrm{P}_{\mathrm{I}}\right\rangle .
$$

Projecting the final state in Eq. (3.4) over $\left|\ell_{\alpha}^{+}, \mathrm{P}_{\mathrm{F}}\right\rangle$ and normalizing, we obtain the flavor neutrino state [10, 49, 9, 50]

$$
\left|\nu_{\alpha}^{\mathrm{P}}\right\rangle=\left(\sum_{i}\left|\mathcal{A}_{\alpha i}^{\mathrm{P}}\right|^{2}\right)^{-1 / 2} \sum_{k} \mathcal{A}_{\alpha k}^{\mathrm{P}}\left|\nu_{k}\right\rangle .
$$

Therefore, a flavor neutrino state is a coherent superposition of massive neutrino states $\left|\nu_{k}\right\rangle$ and the coefficient $\mathcal{A}_{\alpha k}^{\mathrm{P}}$ of the $k^{\text {th }}$ massive neutrino component is given by the amplitude of production of $\nu_{k}$. Since, in general, the amplitudes $\mathcal{A}_{\alpha k}^{\mathrm{P}}$ depend on the production process, a flavor neutrino state depends on the production process. In the following, we will call a flavor neutrino state of the type in Eq. (3.6) a "production flavor neutrino state".

Let us now consider the detection of a flavor neutrino $\nu_{\alpha}$ through the generic chargedcurrent weak interaction process

$$
\nu_{\alpha}+\mathrm{D}_{\mathrm{I}} \rightarrow \mathrm{D}_{\mathrm{F}}+\ell_{\alpha}^{-},
$$

where $D_{I}$ is the target particle and $D_{F}$ represents one or more final particles. In general, since the incoming neutrino state in the detection process is a superposition of massive neutrino states, it may not have a definite flavor. Therefore, we must consider the generic process

$$
\nu+\mathrm{D}_{\mathrm{I}},
$$

with a generic incoming neutrino state $|\nu\rangle$. In this case, the final state of the scattering process is given by

$$
|f\rangle=\mathrm{S}\left|\nu, \mathrm{D}_{\mathrm{I}}\right\rangle
$$

This final state contains all the possible scattering channels:

$$
|f\rangle=\left|\mathrm{D}_{\mathrm{F}}, \ell_{\alpha}^{-}\right\rangle+\ldots,
$$

where we have singled out the scattering channel in Eq. (3.7). We want to find the component

$$
\left|\nu_{\alpha}, \mathrm{D}_{\mathrm{I}}\right\rangle=\sum_{k} \mathcal{A}_{\alpha k}^{\mathrm{D}}\left|\nu_{k}, \mathrm{D}_{\mathrm{I}}\right\rangle
$$

of the initial state $\left|\nu, \mathrm{D}_{\mathrm{I}}\right\rangle$ which corresponds to the flavor $\alpha$, i.e. the component which generates only the scattering channel in Eq. (3.7). This means that $\left|\mathrm{D}_{\mathrm{F}}, \ell_{\alpha}^{-}\right\rangle=\mathrm{S}\left|\nu_{\alpha}, \mathrm{D}_{\mathrm{I}}\right\rangle$. Using the unitarity of the mixing matrix, we obtain

$$
\left|\nu_{\alpha}, \mathrm{D}_{\mathrm{I}}\right\rangle=\mathrm{S}^{\dagger}\left|\mathrm{D}_{\mathrm{F}}, \ell_{\alpha}^{-}\right\rangle .
$$


From Eqs. (3.11) and (3.12), the coefficients $\mathcal{A}_{\alpha k}^{\mathrm{D}}$ are the complex conjugate of the amplitude of detection of $\nu_{k}$ in the detection process in Eq. (3.7):

$$
\mathcal{A}_{\alpha k}^{\mathrm{D}}=\left\langle\nu_{k}, \mathrm{D}_{\mathrm{I}}\left|\mathrm{S}^{\dagger}\right| \mathrm{D}_{\mathrm{F}}, \ell_{\alpha}^{-}\right\rangle .
$$

Projecting $\left|\nu_{\alpha}, \mathrm{D}_{\mathrm{I}}\right\rangle$ over $\left|\mathrm{D}_{\mathrm{I}}\right\rangle$ and normalizing, we finally obtain the flavor neutrino state in the detection process in Eq. (3.7):

$$
\left|\nu_{\alpha}^{\mathrm{D}}\right\rangle=\left(\sum_{i}\left|\mathcal{A}_{\alpha i}^{\mathrm{D}}\right|^{2}\right)^{-1 / 2} \sum_{k} \mathcal{A}_{\alpha k}^{\mathrm{D}}\left|\nu_{k}\right\rangle .
$$

In the following, we will call a flavor neutrino state of this type a "detection flavor neutrino state".

Although the expressions in Eqs. (3.6) and (3.14) for the production and detection flavor neutrino states have the same structure, these states have different meanings. A production flavor neutrino state describes the neutrino created in a charged-current interaction process, which propagates out of a source. Hence, it describes the initial state of a propagating neutrino. A detection flavor neutrino state does not describe a propagating neutrino. It describes the component of the state of a propagating neutrino which can generate a charged lepton with appropriate flavor through a charged-current weak interaction with an appropriate target particle. In other words, the scalar product

$$
A_{\alpha}=\left\langle\nu_{\alpha}^{\mathrm{D}} \mid \nu\right\rangle
$$

is the probability amplitude to find a $\nu_{\alpha}$ by observing the scattering channel in Eq. (3.7) with the scattering process in Eq. (3.8).

In order to understand the connection of the production and detection flavor neutrino states with the standard flavor neutrino states in Eq. (1.1), it is useful to express the $S$-matrix operator as

$$
\mathrm{S}=1-i \int \mathrm{d}^{4} x \mathscr{H}_{\mathrm{CC}}(x),
$$

where we have considered only the first order perturbative contribution of the effective low-energy charged-current weak interaction Hamiltonian

$$
\mathscr{H}_{\mathrm{CC}}(x)=\frac{G_{\mathrm{F}}}{\sqrt{2}} j_{\rho}^{\dagger}(x) j^{\rho}(x)
$$

where $G_{\mathrm{F}}$ is the Fermi constant. The weak charged current $j^{\rho}(x)$ is given by

$$
\begin{aligned}
j^{\rho}(x) & =\sum_{\alpha=e, \mu, \tau} \overline{\nu_{\alpha}}(x) \gamma^{\rho}\left(1-\gamma^{5}\right) \ell_{\alpha}(x)+h^{\rho}(x) \\
& =\sum_{\alpha=e, \mu, \tau} \sum_{k} U_{\alpha k}^{*} \overline{\nu_{k}}(x) \gamma^{\rho}\left(1-\gamma^{5}\right) \ell_{\alpha}(x)+h^{\rho}(x),
\end{aligned}
$$

where $h^{\rho}(x)$ is the hadronic weak charged current. The production and detection amplitudes $\mathcal{A}_{\alpha k}^{\mathrm{P}}$ and $\mathcal{A}_{\alpha k}^{\mathrm{D}}$ can be written as

$$
\mathcal{A}_{\alpha k}^{\mathrm{P}}=U_{\alpha k}^{*} \mathcal{M}_{\alpha k}^{\mathrm{P}}, \quad \mathcal{A}_{\alpha k}^{\mathrm{D}}=U_{\alpha k}^{*} \mathcal{M}_{\alpha k}^{\mathrm{D}},
$$


with the interaction matrix elements

$$
\begin{aligned}
& \mathcal{M}_{\alpha k}^{\mathrm{P}}=-i \frac{G_{\mathrm{F}}}{\sqrt{2}} \int \mathrm{d}^{4} x\left\langle\nu_{k}, \ell_{\alpha}^{+}\left|\overline{\nu_{k}}(x) \gamma^{\rho}\left(1-\gamma^{5}\right) \ell_{\alpha}(x)\right| 0\right\rangle J_{\rho}^{\mathrm{P}_{\mathrm{I}} \rightarrow \mathrm{P}_{\mathrm{F}}}(x), \\
& \mathcal{M}_{\alpha k}^{\mathrm{D}}=i \frac{G_{\mathrm{F}}}{\sqrt{2}} \int \mathrm{d}^{4} x\left\langle\nu_{k}\left|\overline{\nu_{k}}(x) \gamma^{\rho}\left(1-\gamma^{5}\right) \ell_{\alpha}(x)\right| \ell_{\alpha}^{-}\right\rangle J_{\rho}^{\mathrm{D}_{\mathrm{I}} \rightarrow \mathrm{D}_{\mathrm{F}}{ }^{*}}(x) .
\end{aligned}
$$

Here $J_{\rho}^{\mathrm{P}_{\mathrm{I}} \rightarrow \mathrm{P}_{\mathrm{F}}}(x)$ and $J_{\rho}^{\mathrm{D}_{\mathrm{I}} \rightarrow \mathrm{D}_{\mathrm{F}}}(x)$ are, respectively, the matrix elements of the $\mathrm{P}_{\mathrm{I}} \rightarrow \mathrm{P}_{\mathrm{F}}$ and $\mathrm{D}_{\mathrm{I}} \rightarrow \mathrm{D}_{\mathrm{F}}$ transitions.

Using Eq. (3.19), the production and detection flavor neutrino states can be written as

$$
\begin{aligned}
& \left|\nu_{\alpha}^{\mathrm{P}}\right\rangle=\sum_{k} \frac{\mathcal{M}_{\alpha k}^{\mathrm{P}}}{\sqrt{\sum_{j}\left|U_{\alpha j}\right|^{2}\left|\mathcal{M}_{\alpha j}^{\mathrm{P}}\right|^{2}}} U_{\alpha k}^{*}\left|\nu_{k}\right\rangle, \\
& \left|\nu_{\alpha}^{\mathrm{D}}\right\rangle=\sum_{k} \frac{\mathcal{M}_{\alpha k}^{\mathrm{D}}}{\sqrt{\sum_{j}\left|U_{\alpha j}\right|^{2}\left|\mathcal{M}_{\alpha j}^{\mathrm{D}}\right|^{2}}} U_{\alpha k}^{*}\left|\nu_{k}\right\rangle .
\end{aligned}
$$

These states have a structure which is similar to the standard flavor states in Eq. (1.1), with the relative contribution of the massive neutrino $\nu_{k}$ proportional to $U_{\alpha k}^{*}$. The additional factors are due to the dependence of the production and detection processes on the neutrino masses.

In experiments which are not sensitive to the dependence of $\mathcal{M}_{\alpha k}^{\mathrm{P}}$ or $\mathcal{M}_{\alpha k}^{\mathrm{D}}$ on the difference of the neutrino masses it is possible to approximate

$$
\mathcal{M}_{\alpha k}^{\mathrm{P}} \simeq \mathcal{M}_{\alpha}^{\mathrm{P}}, \quad \mathcal{M}_{\alpha k}^{\mathrm{D}} \simeq \mathcal{M}_{\alpha}^{\mathrm{D}} .
$$

In this case, since

$$
\sum_{k}\left|U_{\alpha k}\right|^{2}=1
$$

we obtain, up to an irrelevant phase, the standard flavor neutrino states in Eq. (1.1), which do not depend on the production or detection process. Hence, the standard flavor neutrino states are approximations of the production and detection flavor neutrino states in experiments which are not sensitive to the dependence of the neutrino interaction rate on the difference of the neutrino masses.

In the following section 4 we will show that the correct expressions for the production and detection flavor neutrino states are important in order to be able to describe, in a consistent framework, neutrino oscillations and neutrino production and detection. Then, in the next section 5 we will derive the neutrino oscillation probability starting from the production and detection flavor neutrino states. We will show that, with the appropriate approximations, the oscillation probability reduces to the standard one in Eq. (2.6), which is derived from the approximate flavor neutrino states in Eq. (1.1).

\section{Production and Detection Rates}

In order to measure $\nu_{\alpha} \rightarrow \nu_{\beta}$ oscillations, it is necessary to calculate the neutrino production rate $\Gamma_{\alpha}(E)$ of $\nu_{\alpha}$ in the source and the detection cross section $\sigma_{\beta}(E)$ of $\nu_{\beta}$. The 
number of transition events as a function of the distance $L$ traveled by the neutrino between production and detection and the neutrino energy $E$ is given by

$$
N_{\alpha \beta}(L, E) \propto \Gamma_{\alpha}(E) P_{\nu_{\alpha} \rightarrow \nu_{\beta}}(L, E) \sigma_{\beta}(E),
$$

with a constant of proportionality which depends on the size and composition of the source and detector and on the running time of the experiment. From the measurement of $N_{\alpha \beta}(L, E)$ and the knowledge of $\Gamma_{\alpha}(E)$ and $\sigma_{\beta}(E)$, the experimentalist infers the value of $P_{\nu_{\alpha} \rightarrow \nu_{\beta}}(L, E)$, which gives information on the mixing parameters (elements of the mixing matrix and squared-mass differences) through Eq. (2.6).

Decay rates and cross sections are given by the incoherent sum over the different channels corresponding to different massive neutrinos [51, 52, 53, 54, 55]. The reason is that massive neutrinos are the physical particles which propagate in space-time with definite kinematical properties. However, in section 5 we derived the oscillation probability $P_{\nu_{\alpha} \rightarrow \nu_{\beta}}(L, E)$ starting from the description of neutrinos through flavor states, which are coherent superpositions of massive neutrino states. Then it is natural to ask if the derivation of the oscillation probability is consistent with the calculation of decay rates and cross sections. In the following, we show that the description of neutrinos through flavor states leads to the correct expression for the decay rates and cross sections. Therefore, all quantities in Eq. (4.1) can be calculated in a consistent way.

In order to be definite, we consider the general decay process in Eq. (3.1), in which a flavor neutrino $\nu_{\alpha}$ is produced. Any other process of neutrino production or detection can be treated in an analogous way.

Using the flavor state in Eq. (3.6) and taking into account Eq. (3.5), the amplitude of the general decay process in Eq. (3.1) is given by [50]

$$
\mathcal{A}_{\alpha}^{\mathrm{P}}=\left\langle\nu_{\alpha}^{\mathrm{P}}, \ell_{\alpha}^{+}, \mathrm{P}_{\mathrm{F}}|\mathrm{S}| \mathrm{P}_{\mathrm{I}}\right\rangle=\left(\sum_{i}\left|\mathcal{A}_{\alpha i}^{\mathrm{P}}\right|^{2}\right)^{-1 / 2} \sum_{k} \mathcal{A}_{\alpha k}^{\mathrm{P} *}\left\langle\nu_{k}, \ell_{\alpha}^{+}, \mathrm{P}_{\mathrm{F}}|\mathrm{S}| \mathrm{P}_{\mathrm{I}}\right\rangle=\sqrt{\sum_{i}\left|\mathcal{A}_{\alpha i}^{\mathrm{P}}\right|^{2}} .
$$

Therefore, the decay probability is correctly given by an incoherent sum of the probabilities of production of different massive neutrinos,

$$
\left|\mathcal{A}_{\alpha}^{\mathrm{P}}\right|^{2}=\sum_{i}\left|\mathcal{A}_{\alpha i}^{\mathrm{P}}\right|^{2}
$$

In other words, the coherent character of the flavor state in Eq. (3.6) is irrelevant for the decay probability, which can be obtained either using the flavor neutrino state in Eq. (3.6) or an incoherent mixture of massive neutrino states. The decay rate is then obtained by integrating each massive neutrino contribution to the decay probability over its phase space.

Using the expression in Eq. (3.19) for the amplitude $\mathcal{A}_{\alpha k}^{\mathrm{P}}$, the decay probability in Eq. (4.3) can be written as

$$
\left|\mathcal{A}_{\alpha}^{\mathrm{P}}\right|^{2}=\sum_{k}\left|U_{\alpha k}\right|^{2}\left|\mathcal{M}_{\alpha k}^{\mathrm{P}}\right|^{2},
$$

which is an incoherent sum of the probabilities of production of the different massive neutrinos weighted by $\left|U_{\alpha k}\right|^{2}[51,52,553,54,555]$. 
Therefore, the flavor neutrino state in Eq. (3.6) leads to the correct decay rate for the general decay process in Eq. (3.1). It is clear that this proof can easily be generalized to any charged-current weak interaction process in which flavor neutrinos are produced or detected.

If an experiment is not sensitive to the dependence of $\mathcal{M}_{\alpha k}^{\mathrm{P}}$ on the different neutrino masses, it is possible to use the approximation in Eq. (3.24). In this case, using Eq. (3.25), we obtain

$$
\left|\mathcal{A}_{\alpha}^{\mathrm{P}}\right|^{2}=\left|\mathcal{M}_{\alpha}^{\mathrm{P}}\right|^{2}
$$

If the scale of neutrino masses is negligible in comparison with the experimental resolution, the dependence of $\mathcal{M}_{\alpha}^{\mathrm{P}}$ on the neutrino masses is negligible and the decay probability in Eq. (4.5) reduces to the standard decay probability for massless neutrinos.

The decay probability in Eq. (4.5) can also be obtained starting from the standard flavor states in Eq. (1.1), which are obtained from Eq. (3.6) through the approximation in Eq. (3.24). Indeed, in this case the decay amplitude is given by [56]

$$
\mathcal{A}_{\alpha}^{\mathrm{P}}=\left\langle\nu_{\alpha}, \ell_{\alpha}^{+}, \mathrm{P}_{\mathrm{F}}|\mathrm{S}| \mathrm{P}_{\mathrm{I}}\right\rangle=\sum_{k} U_{\alpha k} \mathcal{A}_{\alpha k}^{\mathrm{P}}=\sum_{k}\left|U_{\alpha k}\right|^{2} \mathcal{M}_{\alpha}^{\mathrm{P}}=\mathcal{M}_{\alpha}^{\mathrm{P}} .
$$

Let us remark, however, that in the case of an experiment which is sensitive to the dependence of $\mathcal{M}_{\alpha k}^{\mathrm{P}}$ on the different neutrino masses, a derivation of the decay amplitude starting from the standard flavor states would lead to an incorrect result. This is due to the fact that in this case the approximation in Eq. (3.24) is not valid and one must take into account the dependence of $\mathcal{M}_{\alpha k}^{\mathrm{P}}$ on the different neutrino masses in the definition of the flavor states.

\section{Neutrino Oscillations}

Let us consider a neutrino oscillation experiment in which $\nu_{\alpha} \rightarrow \nu_{\beta}$ transitions are studied with a production process of the type in Eq. (3.1) and a detection process of the type in Eq. (3.7). In this case, the produced flavor neutrino $\nu_{\alpha}$ is described by the production flavor state $\left|\nu_{\alpha}^{\mathrm{P}}\right\rangle$ in Eq. (3.6). If the neutrino production and detection processes are separated by a space-time interval $(\vec{L}, T)$, the neutrino propagates freely between production and detection, evolving into the state

$$
|\nu(\vec{L}, T)\rangle=e^{-i \mathrm{P}^{0} T+i \overrightarrow{\mathrm{P}} \cdot \vec{L}}\left|\nu_{\alpha}^{\mathrm{P}}\right\rangle,
$$

where $\mathrm{P}^{0}$ and $\overrightarrow{\mathrm{P}}$ are, respectively, the energy and momentum operators. This is the incoming neutrino state in the detection process. The amplitude of the measurable $\nu_{\alpha} \rightarrow$ $\nu_{\beta}$ transitions is given by the scalar product in Eq. (3.15):

$$
A_{\nu_{\alpha} \rightarrow \nu_{\beta}}(\vec{L}, T)=\left\langle\nu_{\beta}^{\mathrm{D}} \mid \nu(\vec{L}, T)\right\rangle=\left\langle\nu_{\beta}^{\mathrm{D}}\left|e^{-i \mathrm{P}^{0} T+i \overrightarrow{\mathrm{P}} \cdot \vec{L}}\right| \nu_{\alpha}^{\mathrm{P}}\right\rangle,
$$

with the detection flavor state $\left|\nu_{\beta}^{\mathrm{D}}\right\rangle$ in Eq. (3.14).

Since the massive neutrinos have definite kinematical properties (energy and momentum), we have, in the plane wave approximation,

$$
\mathrm{P}^{\mu}\left|\nu_{k}\right\rangle=p_{k}^{\mu}\left|\nu_{k}\right\rangle
$$


with

$$
p_{k}^{0}=E_{k}=\sqrt{\left|\vec{p}_{k}\right|^{2}+m_{k}^{2}} .
$$

Using the normalization $\left\langle\nu_{k} \mid \nu_{j}\right\rangle=\delta_{k j}$, we obtain the flavor transition amplitude

$$
A_{\nu_{\alpha} \rightarrow \nu_{\beta}}(\vec{L}, T)=\left(\sum_{i}\left|\mathcal{A}_{\alpha i}^{\mathrm{P}}\right|^{2}\right)^{-1 / 2}\left(\sum_{i}\left|\mathcal{A}_{\beta i}^{\mathrm{D}}\right|^{2}\right)^{-1 / 2} \sum_{k} \mathcal{A}_{\alpha k}^{\mathrm{P}} \mathcal{A}_{\beta k}^{\mathrm{D} *} e^{-i E_{k} T+i \vec{p}_{k} \cdot \vec{L}}
$$

Notice that the consideration of the space-time interval between neutrino production and detection allows one to take into account both the differences in energy and momentum of massive neutrinos [40, 27, 41, 42, 33].

Let us consider the simplest case in which all massive neutrino momenta $\vec{p}_{k}$ are aligned along $\vec{L}$. This assumption is reasonable, because all massive neutrino components are created in the same microscopic production process and detected in the same microscopic detection process, after propagation through the large macroscopic space interval $\vec{L}$. In section $[$ we will show that possible deviations from this assumption do not lead to any observable effect.

In this "one-dimensional approximation", the transition amplitude depends on $L \equiv$ $|\vec{L}|$ and $T$ :

$$
A_{\nu_{\alpha} \rightarrow \nu_{\beta}}(L, T)=\left(\sum_{i}\left|\mathcal{A}_{\alpha i}^{\mathrm{P}}\right|^{2}\right)^{-1 / 2}\left(\sum_{i}\left|\mathcal{A}_{\beta i}^{\mathrm{D}}\right|^{2}\right)^{-1 / 2} \sum_{k} \mathcal{A}_{\alpha k}^{\mathrm{P}} \mathcal{A}_{\beta k}^{\mathrm{D} *} e^{-i E_{k} T+i p_{k} L},
$$

where $p_{k} \equiv\left|\vec{p}_{k}\right|$.

In oscillation experiments in which the neutrino propagation time $T$ is not measured, it is possible to adopt the light-ray $T=L$ approximation, since neutrinos are ultrarelativistic (the effects of possible deviations from $T=L$ are shown to be negligible in section (6). In this case, the phase in Eq. (5.6) becomes

$$
-E_{k} T+p_{k} L=-\left(E_{k}-p_{k}\right) L=-\frac{E_{k}^{2}-p_{k}^{2}}{E_{k}+p_{k}} L=-\frac{m_{k}^{2}}{E_{k}+p_{k}} L \simeq-\frac{m_{k}^{2}}{2 E} L
$$

where $E$ is the neutrino energy neglecting mass contributions. Equation (5.7) shows that the phases of massive neutrinos relevant for the oscillations are independent from the values of the energies and momenta of different massive neutrinos [40, 27, 41, 42, 33], because of the relativistic dispersion relation in Eq. (5.4). In particular, Eq. (5.7) shows that the equal-momentum assumption (A2) in section 2, adopted in the standard derivation of the neutrino oscillation probability, is not necessary in an improved derivation which takes into account both the evolutions in space and in time of the neutrino state.

The probability of $\nu_{\alpha} \rightarrow \nu_{\beta}$ transitions in space is given by

$$
P_{\nu_{\alpha} \rightarrow \nu_{\beta}}(L, E)=\left(\sum_{i}\left|\mathcal{A}_{\alpha i}^{\mathrm{P}}\right|^{2}\right)^{-1}\left(\sum_{i}\left|\mathcal{A}_{\beta i}^{\mathrm{D}}\right|^{2}\right)^{-1} \sum_{k, j} \mathcal{A}_{\alpha k}^{\mathrm{P}} \mathcal{A}_{\beta k}^{\mathrm{D} *} \mathcal{A}_{\alpha j}^{\mathrm{P} *} \mathcal{A}_{\beta j}^{\mathrm{D}} \exp \left(-i \frac{\Delta m_{k j}^{2} L}{2 E}\right)
$$


Using the decomposition in Eq. (3.19), the oscillation probability in Eq. (5.8) can now be written as

$$
\begin{array}{r}
P_{\nu_{\alpha} \rightarrow \nu_{\beta}}(L, E)=\sum_{k, j}\left(\frac{\mathcal{M}_{\alpha k}^{\mathrm{P}} \mathcal{M}_{\alpha j}^{\mathrm{P} *}}{\sum_{i}\left|U_{\alpha i}\right|^{2}\left|\mathcal{M}_{\alpha i}^{\mathrm{P}}\right|^{2}}\right)\left(\frac{\mathcal{M}_{\beta k}^{\mathrm{D} *} \mathcal{M}_{\beta j}^{\mathrm{D}}}{\sum_{i}\left|U_{\beta i}\right|^{2}\left|\mathcal{M}_{\beta i}^{\mathrm{D}}\right|^{2}}\right) \\
\times U_{\alpha k}^{*} U_{\beta k} U_{\alpha j} U_{\beta j}^{*} \exp \left(-i \frac{\Delta m_{k j}^{2} L}{2 E}\right) .
\end{array}
$$

This probability has the same structure as the standard oscillation probability in Eq. (2.6), with additional factors that take into account the effect of the neutrino masses in the production and detection processes. It is clear from Eq. (5.9) that these effects have an influence on the amplitude of the oscillations, but not on the phase, which coincides with the standard one in Eq. (2.6).

Since neutrinos in oscillation experiments are ultra-relativistic and the experiments are not sensitive to the dependence of neutrino interactions on the neutrino masses, the dependence of $\mathcal{M}_{\alpha k}^{\mathrm{P}}$ and $\mathcal{M}_{\beta k}^{\mathrm{D}}$ on the neutrino masses can be neglected, leading to the approximation in Eq. (3.24). In this case, the transition probability in Eq. (5.9) reduces to the standard one in Eq. (2.6), which can be obtained starting from the standard flavor states in Eq. (1.1). As shown in section 3, the standard flavor states are obtained from the production and detection flavor states under the same approximations. Therefore, the standard flavor states are appropriate for the description of neutrino oscillation experiments in the plane wave approximation, as long as the dependence of the production and detection probabilities on the neutrino masses is negligible. These considerations justify the assumption (A1) in section 2, adopted in the standard derivation of the neutrino oscillation probability.

\section{Universality of the Oscillation Phases}

In the previous section 5, we derived the probability of neutrino oscillations under the assumptions that $T=L$ and all massive neutrino momenta $\vec{p}_{k}$ are aligned along $\vec{L}$. In this section we will show that possible deviations from these assumptions do not affect in a significant way the oscillation phases measured in neutrino oscillation experiments, which are correctly given by the standard expression in Eq. (2.6).

The momentum of a massive neutrino created in a production process depends on the characteristics of the interaction, on the nature of the other particles taking part in the process and on the neutrino mass. Let us call $\vec{p}$ and $E=|\vec{p}|$, respectively, the momentum and energy of a massless neutrino. The first order contribution of the neutrino mass to $\vec{p}_{k}$ and $E_{k}$ must be proportional to $m_{k}^{2}$, because the energy-momentum dispersion relation in Eq. (5.4) depends on $m_{k}^{2}$. Therefore, in general, the momentum $\vec{p}_{k}$ can be written to first order in $m_{k}^{2}$ as

where

$$
\vec{p}_{k} \simeq \vec{p}-\vec{\xi} \frac{m_{k}^{2}}{2 E},
$$

$$
\frac{\vec{\xi}}{2 E}=-\left.\frac{\partial \vec{p}_{k}}{\partial m_{k}^{2}}\right|_{m_{k}=0} \text {. }
$$


The value of the vector $\vec{\xi}$ depends on the production process. However, as we will see in the following, the measurable oscillation phases are independent of $\vec{\xi}$. Therefore, they are universal, i.e. independent of the specific nature of the neutrino production process, as well as detection.

In the approximation in Eq. (6.1), the dispersion relation in Eq. (5.4) implies that the energy of $\nu_{k}$ is given by

$$
E_{k} \simeq E+\left(1-\frac{\vec{p} \cdot \vec{\xi}}{E}\right) \frac{m_{k}^{2}}{2 E}
$$

Note that Eq. (6.1) implies that the directions of propagation of different massive neutrinos are slightly different if $\vec{\xi}$ is not collinear with $\vec{p}$. How this is possible can be illustrated in the case of the pion decay production process in Eq. (3.2) as follows. In the rest-frame of the pion, the energy of $\nu_{k}$ can be calculated from energy-momentum conservation to be given by

$$
E_{k}=\frac{m_{\pi}}{2}\left(1-\frac{m_{\mu}^{2}}{m_{\pi}^{2}}\right)+\frac{m_{k}^{2}}{2 m_{\pi}}
$$

where $m_{\pi}$ and $m_{\mu}$ are the masses of the pion and muon, respectively. Since, in the restframe of the pion, the neutrino and the muon are emitted back-to back, all the massive neutrinos are emitted in the same direction. Thus, $\vec{\xi}$ is collinear with $\vec{p}$, leading to

$$
E_{k}=E+(1-\xi) \frac{m_{k}^{2}}{2 E}, \quad p_{k} \equiv\left|\vec{p}_{k}\right| \simeq E-\xi \frac{m_{k}^{2}}{2 E},
$$

with

$$
E=\frac{m_{\pi}}{2}\left(1-\frac{m_{\mu}^{2}}{m_{\pi}^{2}}\right) \simeq 30 \mathrm{MeV}, \quad \xi \equiv|\vec{\xi}|=\frac{1}{2}\left(1+\frac{m_{\mu}^{2}}{m_{\pi}^{2}}\right) \simeq 0.8
$$

Note that the different values of the momenta and energies of different massive neutrinos imply different corresponding values for the momentum and energy of the outgoing muon. Two different massive neutrinos can be produced coherently in the same decay process only if the outgoing muon has energy and momentum uncertainties which are larger, respectively, of the difference of the energies and momenta of the two massive neutrinos. These uncertainties must come from corresponding uncertainties for the pion. Hence, in a rigorous treatment, the pion and the muon must be described by wave packets [34, 35, 31, 36, 37, 38, 39, 32. This implies that also the massive neutrinos must be described by wave packets $[25,26,27,28,29,30,31,32,33$. The spatial extension of the massive neutrino wave packets explains how it is possible that the different massive neutrinos can be detected in the same interaction process even if their space-time trajectories are different.

Let us now consider a boosted reference frame. If the frame is boosted in the direction of the emitted massive neutrinos, obviously they are still collinear. Instead, if the frame is boosted in another direction, collinearity is lost. For example, let us consider a frame boosted with velocity $V$ in a direction which is orthogonal to the neutrino direction in the pion rest frame. In this frame, we have, in an obvious notation,

$$
p_{k \|}^{\prime}=p_{k \|}=p_{k}, \quad p_{k \perp}^{\prime}=-\frac{V}{\sqrt{1-V^{2}}} E_{k} .
$$


Hence, the angle of propagation of $\nu_{k}$ in the boosted frame, with respect to its direction in the rest frame, is given by

$$
\tan \theta_{k}^{\prime}=\frac{p_{k \perp}^{\prime}}{p_{k \|}^{\prime}} \simeq-\frac{V}{\sqrt{1-V^{2}}}\left(1+\frac{m_{k}^{2}}{2 E^{2}}\right) .
$$

Since this angle depends on the neutrino mass, in the boosted frame different massive neutrinos are seen to propagate in slightly different directions. In fact, in this case, $\vec{p}^{\prime}$ and $\vec{\xi}^{\prime}$ are not collinear, since

$$
p_{\|}^{\prime}=p_{\|}=E, \quad p_{\perp}^{\prime}=-\frac{V}{\sqrt{1-V^{2}}} E, \quad \xi_{\|}^{\prime}=\xi_{\|}=\xi, \quad \xi_{\perp}^{\prime}=\frac{V}{\sqrt{1-V^{2}}}(1-\xi)
$$

Since many experiments measure the oscillations of neutrinos produced by pion decay in flight (e.g. atmospheric and accelerator neutrino oscillation experiments), in which the neutrino direction is not constrained to be collinear with the pion direction, in order to be realistic, in this section we consider the general case of not-collinear $\vec{p}$ and $\vec{\xi}$.

Let us now calculate the phases

$$
\phi_{k}=-E_{k} T+\vec{p}_{k} \cdot \vec{L},
$$

in the transition amplitude in Eq. (5.5) considering a deviation of the time $T$ between neutrino production and detection from the light-ray approximation $T=L$ :

$$
T=\frac{L}{|\langle\vec{v}\rangle|}\left(1+\varepsilon_{T}\right)
$$

with the average velocity

$$
\langle\vec{v}\rangle=\frac{1}{N} \sum_{k=1}^{N} \frac{\vec{p}_{k}}{E_{k}} \simeq \frac{\vec{p}}{E}-\left[\frac{\vec{p}}{E}\left(1-\frac{\vec{p} \cdot \vec{\xi}}{E}\right)+\vec{\xi}\right] \frac{\overline{m^{2}}}{2 E^{2}},
$$

where $N$ is the number of massive neutrinos and

$$
\overline{m^{2}}=\frac{1}{N} \sum_{k=1}^{N} m_{k}^{2}
$$

is the average of the squared neutrino masses. The absolute value of the average velocity is given by the usual ultra-relativistic expression

$$
|\langle\vec{v}\rangle| \simeq 1-\frac{\overline{m^{2}}}{2 E^{2}}
$$

In Eq. (6.11), we consider $\varepsilon_{T} \ll 1$, because neutrinos are ultra-relativistic and the deviation from $T=L /|\langle\vec{v}\rangle|$ cannot be larger than the size of the neutrino wave packets, which must be much smaller than $L$ in order to observe the oscillations [26, 27]. To first order in the small ratio $\overline{m^{2}} / E^{2}$, Eq. (6.11) becomes

$$
T \simeq L\left(1+\varepsilon_{T}\right)\left(1+\frac{\overline{m^{2}}}{2 E^{2}}\right) .
$$


Now, we consider also deviations from the assumption that the momenta of all massive neutrinos are aligned along $\vec{L}$. As discussed above, the expression for the neutrino momenta in Eq. (6.1) already implies that, in general, the momenta of different massive neutrinos are not collinear. Moreover, we can consider a deviation from the collinearity of $\vec{L}$ and the average momentum

$$
\langle\vec{p}\rangle=\frac{1}{N} \sum_{k=1}^{N} \vec{p}_{k} \simeq \vec{p}-\vec{\xi} \frac{\overline{m^{2}}}{2 E},
$$

which we can write as

$$
\frac{\langle\vec{p}\rangle}{|\langle\vec{p}\rangle|}=\frac{\vec{L}}{L}+\vec{\varepsilon}_{L}
$$

with $\left|\vec{\varepsilon}_{L}\right| \ll 1$, for the same reasons of $\varepsilon_{T} \ll 1$. At zeroth order in the small ratio $\overline{m^{2}} / E^{2}$, we have

$$
\vec{p} \simeq E\left(\frac{\vec{L}}{L}+\vec{\varepsilon}_{L}\right),
$$

which implies, from Eq. (6.3),

$$
E_{k} \simeq E+\left(1-\frac{\vec{L} \cdot \vec{\xi}}{L}-\vec{\varepsilon}_{L} \cdot \vec{\xi}\right) \frac{m_{k}^{2}}{2 E} .
$$

From Eqs. (6.1), (6.15) and (6.19), for the difference $\Delta \phi_{k j}=\phi_{k}-\phi_{j}$ of the phases in Eq. (6.10) we obtain, at first order in the small quantities $\varepsilon_{T}$ and $\left|\vec{\varepsilon}_{L}\right|$,

$$
\Delta \phi_{k j} \simeq-\frac{\Delta m_{k j}^{2} L}{2 E}+\varepsilon_{k j}
$$

with the contribution

$$
\varepsilon_{k j}=\left[\vec{\varepsilon}_{L} \cdot \vec{\xi}-\varepsilon_{T}\left(1-\frac{\vec{L} \cdot \vec{\xi}}{L}\right)\right] \frac{\Delta m_{k j}^{2} L}{2 E}
$$

in addition to the standard oscillation phase in Eq. (2.6). However, since $\varepsilon_{T} \ll 1$ and $\left|\vec{\varepsilon}_{L}\right| \ll 1$, the contribution $\varepsilon_{k j}$ is non-negligible only for $\Delta m_{k j}^{2} L / 2 E \gg 1$. But in this case oscillations are not measurable, since they are washed out by the average over the energy resolution of the detector (see Ref. [7]). In the case of $\Delta m_{k j}^{2} L / 2 E \sim 1$, in which oscillations are measurable, $\varepsilon_{k j}$ is extremely small and can be safely neglected $\left(e^{i \varepsilon_{k j}} \simeq 1\right)$, leading to the validity of the standard expression in Eq. (2.6) for the oscillation phases.

Note the irrelevance for $\Delta \phi_{k j}$ of the lack of collinearity of the trajectories of different massive neutrinos if $\varepsilon_{T}=\left|\vec{\varepsilon}_{L}\right|=0$. It is due to the fact that the deviation from collinearity of $\nu_{k}$ and $\nu_{j}$ is proportional to $\Delta m_{k j}^{2} / 2 E^{2}$ (see the example in Eq. (6.8)). Thus, it induces in the phase difference $\Delta \phi_{k j}$ effects of higher order in $\Delta m_{k j}^{2} / 2 E^{2}$, which are completely negligible.

In conclusion, in this section we have shown that possible small deviations from the light-ray approximation $T=L$ and from the collinearity of the massive neutrino momenta $\vec{p}_{k}$ and $\vec{L}$ are irrelevant for the measurable oscillation phases, which are universally independent from the specific characteristics of the process of neutrino production, as well as detection. Hence, the time $=$ distance assumption (A3) in section 2, adopted in the standard derivation of the neutrino oscillation probability, is correct. 


\section{Time Average}

In section 5 we derived the probability of flavor neutrino oscillations as a function of the source-detector distance $L$ through the light-ray $T=L$ approximation. In the previous section [6 we have shown that the measurable oscillation phases are stable against possible small deviations from the light-ray approximation. Another way to obtain the oscillation probability in space is through an average of the space-time dependent oscillation probability over the unobserved propagation time $T$ [27],

$$
P_{\nu_{\alpha} \rightarrow \nu_{\beta}}(L) \propto \int \mathrm{d} T P_{\nu_{\alpha} \rightarrow \nu_{\beta}}(L, T)
$$

where the constant of proportionality must be chosen in order to satisfy the conservation of probability constraint

$$
\sum_{\beta} P_{\nu_{\alpha} \rightarrow \nu_{\beta}}(L)=\sum_{\beta} P_{\nu_{\alpha} \rightarrow \nu_{\beta}}(L, T)=1
$$

The time average is done on the oscillation probability, not on the amplitude, since it is an average over the propagation times of different neutrinos in a beam, which contribute incoherently to the oscillation probability.

Let us work, for simplicity, in the approximation in Eq. (3.24) for the production and detection matrix elements. From the one-dimensional approximation of the oscillation amplitude in Eq. (5.6), we obtain the space-time dependent oscillation probability

$$
P_{\nu_{\alpha} \rightarrow \nu_{\beta}}(L, T)=\sum_{k, j} U_{\alpha k}^{*} U_{\beta k} U_{\alpha j} U_{\beta j}^{*} e^{-i\left(E_{k}-E_{j}\right) T+i\left(p_{k}-p_{j}\right) L} .
$$

The integration in Eq. (7.1) leads to

$$
P_{\nu_{\alpha} \rightarrow \nu_{\beta}}(L) \propto \sum_{k, j} U_{\alpha k}^{*} U_{\beta k} U_{\alpha j} U_{\beta j}^{*} e^{i\left(p_{k}-p_{j}\right) L} \delta\left(E_{k}-E_{j}\right) .
$$

Hence, the time average implies that $E_{k}=E_{j}$ [39, 38]. However, from Eq. (6.3) it is clear that in the plane wave approximation this equality is, in general, not possible. In order to allow such an equality, it is necessary to take into account the wave packet character of massive neutrinos, which implies an uncertainty in energy.

In an accurate wave packet treatment, the massive neutrinos are described by superpositions of plane waves with different momenta [26, 27, 28, 29, 30, 31, 32, 33]. Since we are not interested in the wave packet effects, we adopt, for each massive neutrino $\nu_{k}$, an approximate wave packet with a distribution in energy $\phi\left(E_{k}-\widetilde{E}_{k}\right)$, where $\widetilde{E}_{k}$ is the average energy. In this case, the space-time dependent oscillation probability in Eq. (7.3) becomes

$$
\begin{aligned}
P_{\nu_{\alpha} \rightarrow \nu_{\beta}}(L, T)= & \sum_{k, j} U_{\alpha k}^{*} U_{\beta k} U_{\alpha j} U_{\beta j}^{*} \\
& \times \int \mathrm{d} E_{k} \int \mathrm{d} E_{j} \phi\left(E_{k}-\widetilde{E}_{k}\right) \phi^{*}\left(E_{j}-\widetilde{E}_{j}\right) e^{-i\left(E_{k}-E_{j}\right) T+i\left(p_{k}-p_{j}\right) L},
\end{aligned}
$$


with $p_{k}=\sqrt{E_{k}^{2}-m_{k}^{2}}$. The integration over $T$ in Eq. (17.1) leads to

$$
P_{\nu_{\alpha} \rightarrow \nu_{\beta}}(L) \propto \sum_{k, j} U_{\alpha k}^{*} U_{\beta k} U_{\alpha j} U_{\beta j}^{*} \int \mathrm{d} E \phi\left(E-\widetilde{E}_{k}\right) \phi^{*}\left(E-\widetilde{E}_{j}\right) e^{i\left(p_{k}-p_{j}\right) L},
$$

with $p_{k}=\sqrt{E^{2}-m_{k}^{2}}$. In the ultrarelativistic approximation $p_{k} \simeq E-m_{k}^{2} / 2 E$, we obtain

$$
P_{\nu_{\alpha} \rightarrow \nu_{\beta}}(L) \propto \sum_{k, j} U_{\alpha k}^{*} U_{\beta k} U_{\alpha j} U_{\beta j}^{*} \int \mathrm{d} E \phi\left(E-\widetilde{E}_{k}\right) \phi^{*}\left(E-\widetilde{E}_{j}\right) \exp \left(-i \frac{\Delta m_{k j}^{2} L}{2 E}\right) .
$$

Now, we assume that the energy width of the wave packets is much larger than the difference between $\widetilde{E}_{k}$ and $\widetilde{E}_{j}$,

$$
\phi\left(E-\widetilde{E}_{k}\right) \simeq \phi\left(E-\widetilde{E}_{j}\right) \simeq \phi(E-\widetilde{E}),
$$

where $\widetilde{E}$ may be chosen as the average energy of the wave packet of a massless neutrino (or the average among the $\widetilde{E}_{k}$ 's). This is the condition which allows the equality $E_{k}=E_{j}$ without suppressing the oscillation probability. Since the energy width of the wave packets is inversely proportional to their spatial width and the difference $\left|\widetilde{E}_{k}-\widetilde{E}_{j}\right|$ is inversely proportional to the oscillation length 4 , the condition in Eq. (7.8) is verified by spatial wave packets which are much smaller than the oscillation length. This is a necessary condition for the observation of neutrino oscillation which is satisfied in all experiments. Using the approximation in Eq. (17.8), the oscillation probability in Eq. (7.7) becomes

$$
P_{\nu_{\alpha} \rightarrow \nu_{\beta}}(L) \propto \sum_{k, j} U_{\alpha k}^{*} U_{\beta k} U_{\alpha j} U_{\beta j}^{*} \int \mathrm{d} E|\phi(E-\widetilde{E})|^{2} \exp \left(-i \frac{\Delta m_{k j}^{2} L}{2 E}\right) .
$$

Finally, we consider a sharply peaked wave packet, i.e. a wave packet $\phi(E-\widetilde{E})$ with energy uncertainty much smaller than the average energy $\widetilde{E}$. This is a realistic assumption, since in practice the energy uncertainties of the production and detection processes are much smaller than the neutrino energy. In this case, the phase $\Delta m_{k j}^{2} L / 2 E$ is practically constant over the size of the wave packet when the distance $L$ is of the order of the average oscillation length $4 \pi \widetilde{E} / \Delta m_{k j}^{2}$. As we have already remarked in section 6 , this is a necessary condition for the observation of oscillations, since for $L \gg 4 \pi \widetilde{E} / \Delta m_{k j}^{2}$ the oscillations are washed out by the average over the energy resolution of the detector. Hence, using the normalization $\int \mathrm{d} E|\phi(E-\widetilde{E})|^{2}=1$, we finally obtain

$$
P_{\nu_{\alpha} \rightarrow \nu_{\beta}}(L)=\sum_{k, j} U_{\alpha k}^{*} U_{\beta k} U_{\alpha j} U_{\beta j}^{*} \exp \left(-i \frac{\Delta m_{k j}^{2} L}{2 \widetilde{E}}\right) \text {. }
$$

This is the standard oscillation probability in Eq. (2.6), with $\widetilde{E}=E$.

Summarizing, in this section we have shown that the average of the space-time dependent oscillation probability over the unobserved propagation time $T$ leads to the standard oscillation probability in space, taking into account the wave packet character of massive neutrinos, without need of a detailed wave packet model.

${ }^{4}$ Writing $\widetilde{E}_{k} \simeq \widetilde{E}+(1-\xi) m_{k}^{2} / 2 \widetilde{E}$, in analogy with Eq. (6.5), we have $\left|\widetilde{E}_{k}-\widetilde{E}_{j}\right| \propto\left|\Delta m_{k j}^{2}\right| / 2 \widetilde{E} \propto$ $1 / L_{k j}^{\text {osc }}$, where $L_{k j}^{\text {osc }}=4 \pi \widetilde{E} /\left|\Delta m_{k j}^{2}\right|$ is the oscillation length. 


\section{Conclusions}

In this review we have presented a consistent framework for the description of neutrino oscillations and interactions, which is appropriate for the theoretical interpretation of neutrino oscillation experiments, in which neutrinos are produced by a source and detected after propagation over a macroscopic distance. We have shown that the flavor neutrino state that describes a neutrino produced or detected in a charged-current weak interaction process depends on the process under consideration and is appropriate for the description of neutrino oscillations as well as for the calculation of neutrino production and detection rates. A flavor neutrino state can be approximated with the standard expression in Eq. (1.1) only for experiments which are not sensitive to the dependence of neutrino interactions on the different neutrino masses. This is the case of all neutrino oscillation experiments.

We have also reviewed and clarified some subtle points concerning the derivation of the oscillation probability. In particular, we have shown that the oscillation probability can be derived through the usual light-ray time $=$ distance approximation, because possible small deviations have negligible effects on the measurable oscillation phase. We have also shown that the oscillation probability can be derived in an alternative way through an average of the space-time dependent oscillation probability over the unobserved propagation time, taking into account the wave packet character of massive neutrinos, without need of a specific model.

\section{References}

[1] S. Eliezer and A.R. Swift, Nucl. Phys. B105 (1976) 45.

[2] H. Fritzsch and P. Minkowski, Phys. Lett. B62 (1976) 72.

[3] S.M. Bilenky and B. Pontecorvo, Sov. J. Nucl. Phys. 24 (1976) 316.

[4] S.M. Bilenky and B. Pontecorvo, Nuovo Cim. Lett. 17 (1976) 569.

[5] S.M. Bilenky and B. Pontecorvo, Phys. Rept. 41 (1978) 225.

[6] S.M. Bilenky and S.T. Petcov, Rev. Mod. Phys. 59 (1987) 671.

[7] S.M. Bilenky, C. Giunti and W. Grimus, Prog. Part. Nucl. Phys. 43 (1999) 1, hep-ph/9812360.

[8] M. Gonzalez-Garcia and Y. Nir, Rev. Mod. Phys. 75 (2003) 345, hep-ph/0202058.

[9] W.M. Alberico and S.M. Bilenky, Phys. Part. Nucl. 35 (2004) 297, hep-ph/0306239.

[10] C. Giunti, C.W. Kim and U.W. Lee, Phys. Rev. D45 (1992) 2414.

[11] M. Blasone and G. Vitiello, Ann. Phys. 244 (1995) 283, hep-ph/9501263.

[12] M. Blasone, P.A. Henning and G. Vitiello, Phys. Lett. B451 (1999) 140, hep-th/9803157. 
[13] K. Fujii, C. Habe and T. Yabuki, Phys. Rev. D59 (1999) 113003, hep-ph/9807266.

[14] M. Blasone and G. Vitiello, Phys. Rev. D60 (1999) 111302, hep-ph/9907382.

[15] K. Fujii, C. Habe and T. Yabuki, Phys. Rev. D64 (2001) 013011, hep-ph/0102001.

[16] M. Blasone, A. Capolupo and G. Vitiello, Phys. Rev. D66 (2002) 025033, hep-th/0204184.

[17] M. Blasone, P.P. Pacheco and H.W.C. Tseung, Phys. Rev. D67 (2003) 073011, hep-ph/0212402.

[18] C.R. Ji and Y. Mishchenko, Phys. Rev. D65 (2002) 096015, hep-ph/0201188.

[19] A. Capolupo, (2004), hep-th/0408228.

[20] M. Blasone et al., Phys. Rev. D72 (2005) 013003, hep-ph/0505178.

[21] C. Giunti, Eur. Phys. J. C (2005), hep-ph/0312256.

[22] Y. Li and Q. Liu, (2006), hep-ph/0604069.

[23] L. Wolfenstein, Phys. Rev. D17 (1978) 2369.

[24] S.P. Mikheev and A.Y. Smirnov, Sov. J. Nucl. Phys. 42 (1985) 913.

[25] S. Nussinov, Phys. Lett. B63 (1976) 201.

[26] B. Kayser, Phys. Rev. D24 (1981) 110.

[27] C. Giunti, C.W. Kim and U.W. Lee, Phys. Rev. D44 (1991) 3635.

[28] K. Kiers, S. Nussinov and N. Weiss, Phys. Rev. D53 (1996) 537, hep-ph/9506271.

[29] K. Kiers and N. Weiss, Phys. Rev. D57 (1998) 3091, hep-ph/9710289.

[30] C. Giunti and C.W. Kim, Phys. Rev. D58 (1998) 017301, hep-ph/9711363.

[31] M. Zralek, Acta Phys. Polon. B29 (1998) 3925, hep-ph/9810543.

[32] C. Giunti, JHEP 11 (2002) 017, hep-ph/0205014.

[33] C. Giunti, Found. Phys. Lett. 17 (2004) 103, hep-ph/0302026.

[34] C. Giunti et al., Phys. Rev. D48 (1993) 4310, hep-ph/9305276.

[35] C. Giunti, C.W. Kim and U.W. Lee, Phys. Lett. B421 (1998) 237, hep-ph/9709494.

[36] M. Nauenberg, Phys. Lett. B447 (1999) 23, hep-ph/9812441.

[37] C.Y. Cardall, Phys. Rev. D61 (2000) 073006, hep-ph/9909332.

[38] M. Beuthe, Phys. Rept. 375 (2003) 105, hep-ph/0109119.

[39] M. Beuthe, Phys. Rev. D66 (2002) 013003, hep-ph/0202068. 
[40] R.G. Winter, Lett. Nuovo Cim. 30 (1981) 101.

[41] C. Giunti and C.W. Kim, Found. Phys. Lett. 14 (2001) 213, hep-ph/0011074.

[42] C. Giunti, Mod. Phys. Lett. A16 (2001) 2363, hep-ph/0104148.

[43] S.M. Bilenky et al., Phys. Rept. 379 (2003) 69, hep-ph/0211462.

[44] C. Giunti and M. Laveder, (2003), hep-ph/0310238, In 'Developments in Quantum Physics - 2004', p. 197-254, edited by F. Columbus and V. Krasnoholovets, Nova Science Publishers, Inc.

[45] S.M. Bilenky and M.D. Mateev, (2006), hep-ph/0604044.

[46] Y. Grossman and H.J. Lipkin, Phys. Rev. D55 (1997) 2760, hep-ph/9607201.

[47] L. Stodolsky, Phys. Rev. D58 (1998) 036006, hep-ph/9802387.

[48] H.J. Lipkin, Phys. Lett. B579 (2004) 355, hep-ph/0304187.

[49] S.M. Bilenky and C. Giunti, Int. J. Mod. Phys. A16 (2001) 3931, hep-ph/0102320.

[50] C. Giunti, (2004), hep-ph/0402217.

[51] R.E. Shrock, Phys. Lett. B96 (1980) 159.

[52] B.H.J. McKellar, Phys. Lett. B97 (1980) 93.

[53] I.Y. Kobzarev et al., Sov. J. Nucl. Phys. 32 (1980) 823.

[54] R.E. Shrock, Phys. Rev. D24 (1981) 1232.

[55] R.E. Shrock, Phys. Rev. D24 (1981) 1275.

[56] C. Giunti, (2003), hep-ph/0302045. 